POLAR SYSTEMS 


\title{
MAGNETIC FIELDS AND ACCRETION STREAMS IN POLARS
}

\author{
AXEL D. SCHWOPE \\ Astrophysical Institute Potsdam, An der Sternwarte 16, \\ 14482 Potsdam, Germany. (ASchwope@aip.de)
}

\begin{abstract}
The flow of matter, the accretion geometry and the energy release in polars (AM Her binaries) are to a large extent dominated by the strong magnetic field of the accreting white dwarf. In this review I summarize the main tools used to investigate the magnetic fields and the accretion streams, using optical and X-ray spectroscopy and photometry.
\end{abstract}

\section{Magnetic fields in polars}

Cyclotron Radiation. The optical continua of polars in their high accretion states are fed by a strong cyclotron source at the footpoints of accreting field lines. These regions, of size $f \sim 10^{-4}$ (fraction of the white dwarf surface), are heated by hydrodynamic shocks or by particle bombardment to temperatures of a few $\mathrm{keV}$. The ionized plasma is forced to emit electromagnetic radiation on the cyclotron fundamental and in the overtones. Depending on plasma temperature, magnetic field strength and field orientation, resolvable cyclotron harmonics lie in the optical window between 3500 and $10000 \AA$ and can be used in order to infer the field.

Historically, the first direct observational evidence for the presence of a magnetic compact star in AM Her was derived from strong optical polarization (Tapia 1977) and a field strength in excess of $10^{8} \mathrm{G}$ (100 MG) was estimated on the assumption that the observed radiation corresponds to the cyclotron fundamental. The first genuine measurement of the field strength in a polar was possible after identification of cyclotron harmonics in spectra of VV Pup (Visvanathan \& Wickramasinghe 1979; Wickramasinghe \& Meggitt 1982). Meanwhile, this kind of measurement has been done for a number of systems and the scientific trade-off is rather large. The necessary prerequisite in order to do cyclotron spectroscopy is access to a medium- or large-sized telescope equipped with a low-resolution op- 


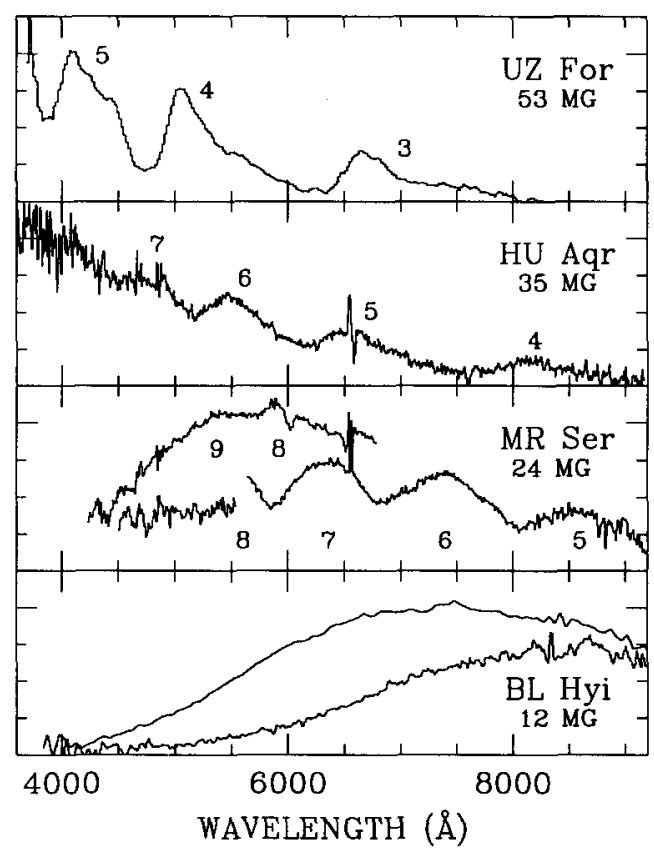

Figure 1. Collection of cyclotron spectra of AM Her binaries (polars) with different field strengths. The spectra in different panels were scaled arbitrarily, the low- and high-state spectra shown for BL $\mathrm{Hyi}$ and $\mathrm{MR}$ Ser, respectively, were scaled by factors of 20 and 2 with respect to each other. Integer numbers designate identified cyclotron harmonics.

tical and/or infrared spectrograph, coverage of preferably at least one orbital period $(80 \ldots 480 \mathrm{~m})$ of the object concerned, and some co-operation of the target regarding e.g. brightness. Although in some cases cyclotron humps are obvious even in the raw spectra, thus allowing a field measurement, it is necessary to correct for other sources of light (stellar continua, recombination radiation, second accretion region) in order to uncover genuine cyclotron spectra. Results of this procedure for a number of systems sorted according to field strength are shown in Fig. 1. The current census of field measurements is compiled in Table 1. All the spectra shown were extracted at binary phases which correspond to large angles, $70^{\circ} \ldots 90^{\circ}$, between the observer and the local magnetic field in the accretion spot. The main features of the observed cyclotron spectra are compatible with basic cyclotron theory (Wickramasinghe \& Meggitt 1985; Schwope 1990). The occurrence of resolvable cyclotron humps is restricted to low harmonic numbers $m_{\max } \leq 10$, large viewing angles $\gtrsim 45^{\circ}$, and relatively low temperatures $5 \ldots 10 \mathrm{keV}$ (compared with the adiabatic shock temperature). If the field is low, as in the case of BL Hyi (Schwope, Beuermann \& Jordan 1995a), the harmonic range covered by optical observations is between $m_{\mathrm{H}}=10$ and 22 . The harmonics are then spaced so closely and the overlap is so large that they are smeared to a smooth quasi-continuum; the field measurement is then possible only using either Zeeman lines or by cyclo- 
tron spectroscopy in the infrared. This has been exercised for the cases of AM Her and ST LMi by Bailey, Ferrario \& Wickramasinge (1991) and Ferrario, Bailey \& Wickramasinge (1993). The absence of optical cyclotron lines, the red optical continua and the occasional detection of halo Zeeman lines in a low field make BL Hyi, WW Hor, EF Eri and RXJ1957-57 suitable future targets for cyclotron spectroscopy in the infrared.

If the field is sufficiently high, and overlap of harmonics not too large, their detection is considerably facilitated if a specific system is observed at reduced accretion rate (e.g. MR Ser, second panel in Fig. 1). Then neither a high temperature nor the spread in angle, temperature, particle density and/or magnetic field are large enough to dilute the cyclotron line pattern. For high-field polars (as HU Aqr and UZ For, upper panels in Fig. 1) the detection of cyclotron lines becomes obviously possible, independent of accretion rate (brightness).

The overall shape of the cyclotron spectra suggest that the bulk of emission is shifted from long to short wavelengths with increasing field strength (an additional but smaller blue-shift within one system results from enhanced accretion). This may change our perception of ultraviolet spectra of high-field polars. It appears possible that a major fraction of the cyclotron flux is released in the ultraviolet regime (a never-confirmed prediction of the basic model of Lamb \& Masters 1979) and it seems promising to detect cyclotron lines in low-resolution UV spectra of e.g. UZ For, VV Pup (second pole), RXJ0515 and QS Tel (RXJ1938). It also appears likely that at long last the pieces of the energetic puzzle in AM Hers can be put together and that the role of the magnetic field will be understood. Recent steps ahead on the observational side have been undertaken e.g. by Beuermann \& Schwope (1994) who have shown that the hard X-ray bremsstrahlung in ROSAT spectra is systematically suppressed in high-field polars (Fig. 2). Woelk \& Beuermann (1996) (see also Beuermann \& Woelk 1996) have investigated theoretically the relation between maximum electron temperature, thickness of the emission region, magnetic field strength and specific mass accretion rate, by solving the stationary radiation hydrodynamics including cyclotron absorption. The behaviour of the observed cyclotron spectra can be described by simply assuming a homogeneous emission region which is characterized for a given field strength and viewing angle by a single size parameter $\Lambda=n_{\mathrm{e}} s / B\left(n_{\mathrm{e}}=\right.$ electron density, $s=$ geometrical path length, $B=$ field strength). It essentially determines the switchover frequency from optically thick to thin radiation (Wickramasinghe \& Meggitt 1985). Its dependence on field strength for a couple of systems is displayed in Fig. 2. From there it seems to be evident that a strong correlation exists between $\Lambda$ and $B$ in a sense predicted by Woelk \& Beuermann (1996: collapse of the size of the emission region with increased cyclotron efficiency). The size 


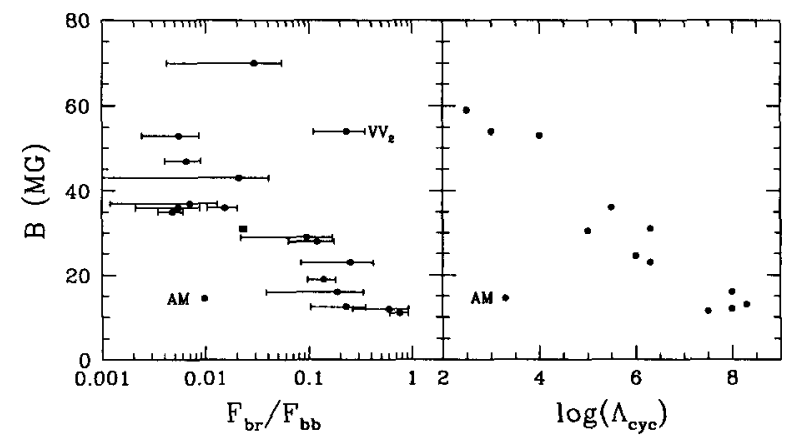

Figure 2. Soft X-ray excess in the ROSAT band (left panel, adapted from Beuermann \& Schwope 1994) and size parameter of cyclotron spectra (right panel) for emission regions in polars as a function of field strength.

of this effect, however, is much larger ( $\sim 5$ decades) than the theoretical prediction ( $\sim 2$ decades). This is a hint of a biased determination of $\Lambda$ in the presence of pronounced cyclotron lines. An extreme example supporting this view is $\mathrm{AM} H \mathrm{Her}$, whose optical radiation peaks at $\sim 8000 \AA$, suggesting a high $\log \Lambda \sim 7$ (no optical cyclotron lines), whereas a fit to the resolved low harmonics in the infrared yields the surprisingly low value of $210^{3}$. The same may apply to the high-field regions in UZ For (main pole), VV Pup, and DP Leo (second poles), where only the low-harmonic optical tail was observed. Summing up, recent observational and theoretical progress are in qualitative agreement. A detailed quantitative comparison is difficult at present due to the lack of sufficient coverage of the cyclotron spectra and to the one-dimensionality of the observational approach (white dwarf mass and specific mass accretion rate $\dot{M}$ must be included).

Another facet of cyclotron spectroscopy is its potential to determine the accretion geometry from the observed motion of cyclotron harmonics. Once the field strength and temperature are fixed, the wavelength of a cyclotron line is completely determined by the viewing angle $\Theta$, which itself is a combination of $i, \delta$ and $\phi$ (orbital inclination, field line inclination, and phase angle). Thus cyclotron spectroscopy can substitute for photoelectric polarimetry (although presently with less time resolution) and recent examples for full-phase tracing of the moving cyclotron pattern can be found in Schwope et al. (1995b) and Burwitz et al. (1996) for QS Tel and RXJ0453.

Zeeman Spectroscopy. In Fig. 3 normalized spectra of MR Ser obtained in high and low accretion states (adapted from Schwope et al. 1993b) are shown. They highlight two different situations where Zeeman-shifted absorption can potentially be observed in polars, here as a very rare case in one system. In the high state (top panel) narrow Zeeman absorption lines are seen against the bright cyclotron background, hence they are clearly of non-photospheric origin. The field strength deduced from the observed 


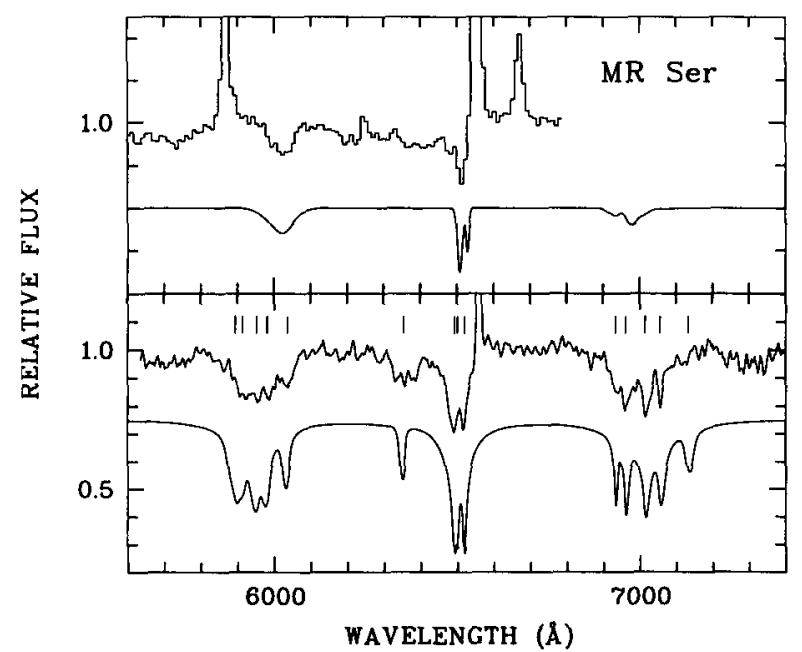

Figure 3. Normalized Zeeman spectra of MR Ser detected in high (top) and low states (bottom), respectively. In the high state the $\mathrm{H} \alpha$ Zeeman triplet is seen against the bright cyclotron background (halo absorption, cyclotron humps at $5950 \AA$ and $6700 \AA$ ), in the low state the Zeeman spectrum originates in the white dwarfs photosphere. Model curves below the spectra are explained in the text.

Zeeman split is $24.5 \mathrm{MG}$ with a spread $\sigma=1 \mathrm{MG}$ (Gaussian distribution assumed; fit below the observed spectrum). The field strength is the same as that deduced from the cyclotron humps. Obviously, cool absorbing matter can coexist with the hot emission plasma. In the low state, when cyclotron radiation from the spot and recombination radiation from the stream are both negligible, the observed spectra are dominated by the stellar photospheres. The white-dwarf's Zeeman-spectrum, which appears after subtraction of a template M-star spectrum, is the flux-weighted average over the visible hemisphere of the white dwarf. Attempts to fit the observed spectra of polars in general, and that of MR Ser in particular, with centered dipolar models regularly fail, either because the motion of Zeeman lines at different magnetic phase is not reflected by the simple models (see e.g. Ferrario et al. 1992, Schwope et al. 1995a for the cases of V834 Cen and BL Hyi) or because the observed Zeeman lines are narrower than predicted (the most striking case is that of MR Ser, where the complete set of non-degenerate Zeeman lines of $\mathrm{H} \alpha$ could be resolved). Models applied so far therefore assume either shifted dipoles, shifted mostly along the $z$ axis, or dipole-quadrupole combinations (' $D+Q$ ' in Table 1). The effect of both refinements is that the field strength on one hemisphere becomes more homogeneous with respect to the centered dipole, which reduces both the motion as well the width of the lines (the curve below the low-state Zeeman spectrum of MR Ser is a white-dwarf model calculated for $T_{\text {eff }}=10000 \mathrm{~K}$, $B_{\text {pole }}=59 \mathrm{MG}, z_{\text {off }}=0.3 \mathrm{R}_{\mathrm{wd}}$ ), where $z_{\text {off }}$ is the off-centering of the dipole along the symmetry axis. 
TABLE 1. Field strengths of white dwarfs in polars. The field strengths refer to cyclotron emission line regions at the main $\left(B_{\text {cyc1 } 1}\right)$ and the secondary $\left(B_{\text {cyc2 }}\right)$ accretion region, the accretion halo $\left(B_{\mathrm{h}}\right)$, and the mean or effective, i.e. flux-weighted, photospheric field $\left(B_{\mathrm{ph}}\right)$. This is an update of Schwope (1995), where a complete reference list is given. Here only references which were not included in the previous edition, or which have changed in the meantime, are given. The systems are sorted according to decreasing field strength of the centered-dipole equivalent.

\begin{tabular}{|c|c|c|c|c|c|}
\hline Object & $B_{\text {cyc1 }}$ & $B_{\text {cyc2 }}$ & $B_{\mathrm{h}}$ & $B_{\mathrm{ph}}$ & Ref./Comment \\
\hline RXJ0515+01 & 62 & & & & Ref. 1 \\
\hline QS Tel (RXJ1938-46) & 47 & $70(80)$ & & & Ref. $2, z_{\mathrm{off}}=0.1$ \\
\hline UZ For & 53 & & & & \\
\hline MR Ser & $24 \ldots 25$ & & 24.5 & 27.3 & $z_{\text {off }}=0.3$ \\
\hline RXJ1149+28 & 43 & & & & \\
\hline DP Leo & $30 . \ldots 32$ & 59 & 29 & & \\
\hline VV Pup & $30 \ldots 31.5$ & $54 \ldots 56$ & & & \\
\hline EU Cnc & 42: & & & & \\
\hline RXJ0453-42 & 36 & & & & Ref. 3 \\
\hline HU Aqr (RXJ2107-05) & 35 & & & & \\
\hline $\mathrm{RE} 1307+535$ & 35: & & & & Ref. 4 \\
\hline EK UMa & $35: / 47:$ & & & & \\
\hline QQ Vul & $31: / 36:$ & & & & \\
\hline V834 Cen & 23 & & 23 & 22 & $z_{\mathrm{off}}=0.1$ \\
\hline BL Hyi & & & 12 & 22 & Ref. $5, D+Q$ \\
\hline AN UMa & 29 & & & & \\
\hline BY Cam & 28 & & & & \\
\hline AM Her & 14.5 & & & 13 & $z_{\text {off }}=0.17$ \\
\hline RXJ0531-46 & 19 & & & & \\
\hline ST LMi & 12 & & & 19 & \\
\hline $\mathrm{RXJ1957-57}$ & & & 16 & & Ref. 6 \\
\hline EF Eri & & & 13 & & \\
\hline V2301 Oph $(1 \mathrm{H} 1752+08)$ & & & & 7 & Ref. 7 \\
\hline
\end{tabular}

REFERENCES: (1) Shafter et al. 1995, ApJ 443, 419; (2) Schwope et al. 1995b; (3) Burwitz et al. 1996, A\&A; (4) Osborne et al. 1994, MNRAS 270, 650; (5) Schwope et al. 1995a; (6) Thomas et al. 1996, A\&A, in press; (7) Ferrario et al. 1995, MNRAS 273, 17

The Census. In Table $1 \mathrm{I}$ have collected all available measurements of magnetic fields in polars. It contains 23 systems, hence a fraction of $\sim 50 \%$ of all known systems. It is therefore probably only a question of telescope time before more field measurements will be available in binary white dwarfs than in single ones [currently 41 known (Schmidt 1995)]. The latter cover three decades in field strength, the polar WDs only one ( $7 \ldots 70 \mathrm{MG})$. At low fields they lose synchronism and the AM Her phenomenon vanishes, 
whereas the ultimate reason for the high-field cutoff is still a matter of debate. One hypothesis is that high-field systems evolve so fast that their detection probability becomes very low (Hameury, King \& Lasota 1988). Wickramasinghe \& Wu (1994) on the other hand suspected that high fields prevent the evolution of these systems in general.

Cyclotron spectroscopy is by far the most succesful method of measuring field strengths in polars; it provides about $2 / 3$ of all entries in Table 1 . The numbers given for BY Cam and AN UMa are revised compared to the original publications (Cropper et al. 1989). This became necessary through the application of full spectral models (Schwope 1991) instead of an analytic formula describing the position of individual cyclotron humps.

In all three cases where both cyclotron emission and halo Zeeman absorption lines have been observed the field strengths agree, thus qualifying halo Zeeman lines in general as indicators of the field in the accretion region, even in those cases where no cyclotron lines are detected. Three confirmed polars exhibit two systems of cyclotron lines originating from a main (X-ray bright) and a secondary accretion region with, in all cases, very different field strengths (Table 1), which is a strong hint of the presence of non-dipolar field structures on the white dwarf. This is supported also by Zeeman spectroscopy, which has been applied in detail to six AM Her binaries. In no case was a successful fit achieved using centered dipole models, significantly decentered dipoles or dipole-quadrupole configurations were always required to reflect the data.

\section{Accretion streams}

Being the vehicles transporting matter from the red companion star to the accreting white dwarf, the accretion streams did not attract so much attention, either theoretically or observationally. Their physics is quite complex and their state subject to several plasma-physical instabilities, most of which are discussed by Hameury, King \& Lasota (1986). Observationally they manifest themselves in each polar by strong, asymmetric and variable emission lines (mainly $\mathrm{H}, \mathrm{He}$ I and $\mathrm{He} \mathrm{II}$ ), and by so-called absorption dips observed at IR, optical and X-ray wavelengths in systems with a high enough inclination (provided observer and accretion spot are on the same hemisphere). In these cases the out-of-plane part of the accretion stream (vertical stream) crosses the line of sight towards the accretion spot and causes absorption features (photoelectric absorption at X-ray wavelengths, free-free or grey absorption in the optical). An overview of relevant data and analysis of the dips was given recently by Watson (1995). A third possibility to investigate the properties of the stream are the light curves of eclipsing systems where the stream emerges as a distinct source of light 

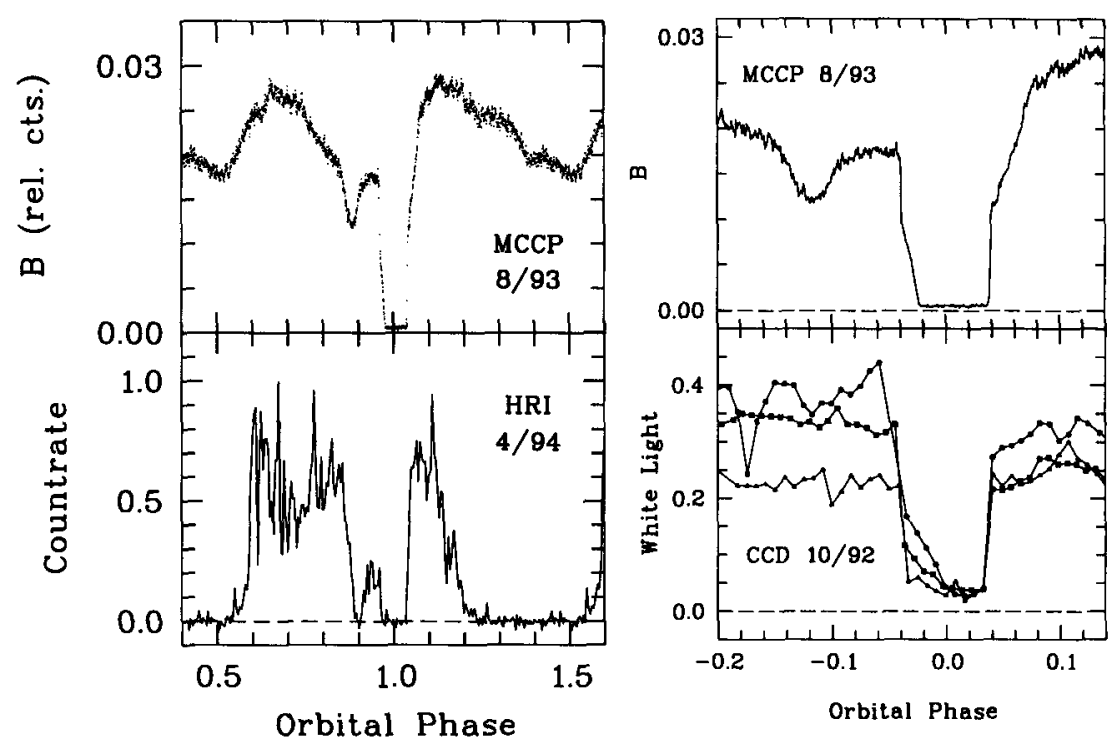

Figure 4. Optical and X-ray light curves of HU Aqr optained at high and intermediate (lower left) accretion states. Left: phase-folded ROSAT HRI and optical $B$-band light curves in overview; right: optical $B$-band (phase-folded) and white-light measurements (three original time sequences) on an expanded scale around eclipse.

during ingress and egress. A good example for the case of UZ For using HST data can be found in Schmidt et al. (1993).

The analysis of emission line data to yield definite statements about the stream has been hampered so far, mainly by lack of data at sufficient time resolution. For the remainder of this Section I will discuss a unique example among the AM Her binaries, HU Aqr (= RX J2107.9-0518), which displays all the features mentioned: an extended eclipse with a bright accretion stream, a pronounced pre-eclipse dip at optical and X-ray wavelengths and a distinct fingerprint of the accretion stream in trailed high-resolution spectrograms. It may serve as a template object to reach a deeper understanding of many of the spectroscopic data brought together in the literature for the different objects. For further reading on HU Aqr see Schwope et al. (1993a, 1995c), Glenn et al. (1994), Hakala (1995), Schwope (1995).

In Fig. 4 I show optical and X-ray light curves of HU Aqr obtained on different occasions in states of high and intermediate accretion. In Fig. 5 a trailed spectrogram of the spectral region around the He II 4686 line with high time and spectral resolution is shown (phase resolution 0.01 of the $125 \mathrm{~m}$ binary) as well as its transformation to velocity space (Doppler tomogram). The high-speed photometry and spectroscopy of Figs. 4 and 5 

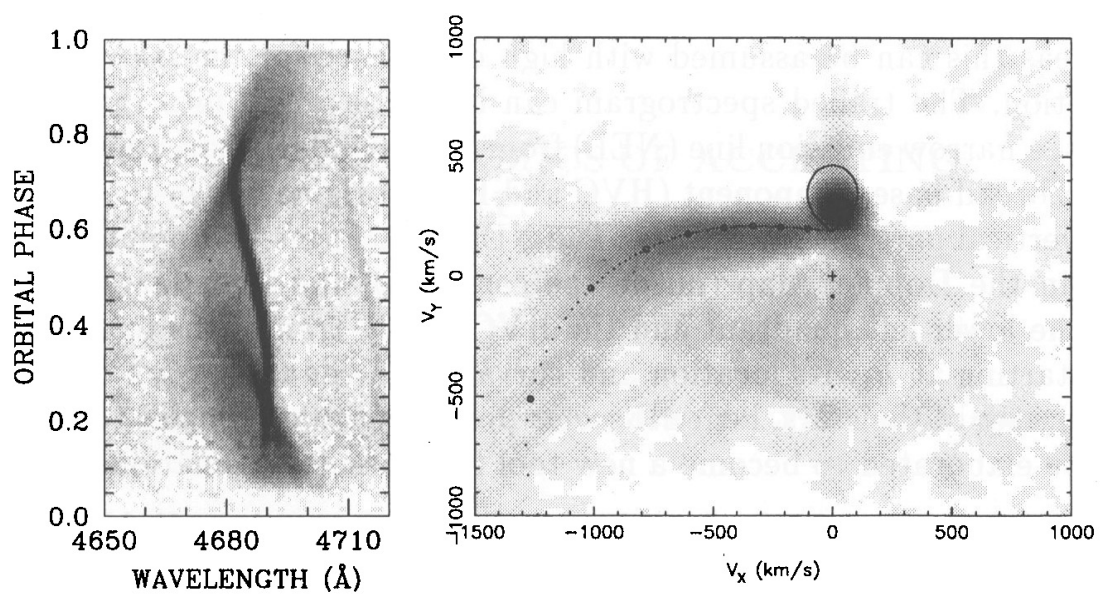

Figure 5. HU Aqr. Left: phase-folded continuum-subtracted spectrogram of He II 4686. Right: Doppler map of He II 4686 calculated by filtered back-projection. The overlay shows the secondary star and the horizontal stream for an assumed velocities $K_{1}=85 \mathrm{~km} \mathrm{~s}^{-1}$, $K_{2}=340 \mathrm{~km} \mathrm{~s}^{-1}$.

were obtained simultaneously. Since HU Aqr is a high-inclination system, $i \simeq 85^{\circ}$, the phase of the absorption dip prior to eclipse indicates the location of the stagnation region and its width gives the size of the stagnation region in the orbital plane. The phase of mid-dip is somewhat variable, $43^{\circ}$ in 1993 August and $35^{\circ}$ in 1994 April, respectively. Its shape in the optical is well described by a Gaussian curve. The X-ray eclipse at dip-phase is total, the X-ray flux between the dip and the genuine eclipse around phase $\phi=0.0$ is reduced with respect to pre-dip or post-eclipse phases, indicating the presence of some absorbing matter above the whole horizontal stream between the coupling region and the $L_{1}$ point. At optical wavelengths only the cyclotron source is obscured at dip-phase, the remaining light is the bright accretion stream. It appears as a prominent and distinct source of light also at eclipse ingress and egress. The optical eclipse reveals the presence of two sources of light, the small accretion spot (cyclotron radiation) which causes the steep initial ingress and egress $\left(\Delta t_{\mathrm{i}, \mathrm{e}} \simeq 3 \mathrm{~s}\right)$ and the accretion stream which is much more extended. Apart from the back-and-forth motion of the coupling region which derives from the variable phase of dip-center, HU Aqr displays episodes with completely different appearance (Fig. 4, bottom right). Then no distinct and well-shaped (macro-) dips can be detected (but perhaps there are several micro-dips at arbitrary phase prior to eclipse) and ingress/egress times of the stream are much longer than in a mode of normal accretion (top right). It is not clear at all whether the stream in this anomalous mode of accretion is bound to a ballistic trajec- 
tory in its horizontal part and to e.g. dipolar field lines in its vertical part, whereas this can be assumed with high confidence for the state of regular accretion. The trailed spectrogram can be deconvolved into three components, a narrow emission line (NEL) from the secondary star, a high-velocity and a broad base component ( $\mathrm{HVC}$ and $\mathrm{BBC}$ ) originating in the horizontal and vertical parts of the stream. The NEL transforms to a point-like structure in the Doppler map (inside the contour of the secondary), the BBC fills the lower left quadrant and the HVC finally transforms to a cometary tail starting at $L_{1}$. Its location and curvature in the tomogram depends on the masses of the stars, hence it seems promising that Doppler tomography can be elaborated to become a new tool for mass determinations in polars.

Acknowledgements. I acknowledge collaboration with K.-H. Mantel (Munich), K. Horne (St. Andrews), S. Jordan (Kiel), H.-C. Thomas (MPA Garching) and K. Beuermann (Göttingen). This work has been supported by the BMFT under grant 50 OR 94035 .

\section{References}

Bailey, J., Ferrario, L., Wickramasinghe, D.T., 1991, MNRAS, 251, 37P

Beuermann, K., Schwope, A.D., 1994, in "Interacting Binary Stars", ed. A. Shafter, ASP Conf. Ser. 56, 119

Beuermann, K., Woelk, U., 1996, these proceedings, p199

Burwitz, V., Reinsch, K., Schwope, A.D., et al., 1996, A\&A, 305, 507

Cropper, M., Mason, K.O., Branduardi-Raymont, G., et al., 1989, MNRAS, 236, 29P

Ferrario, L., Bailey, J., Wickramasinghe, D.T., 1993, MNRAS, 262, 285

Ferrario, L., Wickramasinghe, D.T., Bailey, J., et al., 1992, MNRAS, 256, 252

Glenn, J., Howell, S.B., Schmidt, G.D., et al., 1994, Ap. J., 424, 967

Hakala, P.J., 1995, A\&A, 296, 164

Hameury, J.-M., King, A.R., Lasota, J.-P., 1986, MNRAS, 218, 695

Hameury, J.-M., King, A.R., Lasota, J.-P., 1988, MNRAS, 237, 848

Lamb, D. Q., Masters, A.R., 1979, Ap. J., 234, L117

Schmidt, G., 1995, Rev. in Mod. Astr., 8, 147

Schmidt, G., Liebert, J., Stockman, H., Holberg, J., 1993, Ann. Isr. Phys. Soc., 10, 13

Schwope, A.D., 1990, Rev. in Mod. Astr., 3, 44

Schwope, A.D, 1991, PhD thesis, TU Berlin

Schwope, A.D., 1995, Rev. in Mod. Astr., 8, 125

Schwope, A.D., Beuermann, K., Jordan, S., 1995a, A\&A, 301, 447

Schwope, A.D., Thomas, H.-C., Beuermann, K., 1993a, A\&A, 271, L25

Schwope, A.D., Beuermann, K., Jordan, S., Thomas, H.-C., 1993b, A\&A, 278, 498

Schwope, A.D., Schwarz, R., Mantel, K.-H., et al., 1995c, in "Magnetic Cataclysmic Variables", eds D. Buckley, B. Warner, ASP Conf. Ser. 85, 166

Schwope, A.D. et al., 1995b, A\&A, 293, 764

Tapia, S., 1977, Ap. J., 212, L125

Visvanathan, N., Wickramasinghe, D.T., 1979, Nature, 281, 47

Watson, M.G., 1995, in "Magnetic Cataclysmic Variables", eds D. Buckley, B. Warner, ASP Conf. Ser. 85, 179

Wickramasinghe, D.T., Meggitt, S.M.A., 1982, MNRAS, 198, 975

Wickramasinghe, D.T., Meggitt, S.M.A., 1985, MNRAS, 214, 605

Wickramasinghe, D.T., Wu, K., 1994, MNRAS, 266, L1

Woelk, U., Beuermann, K., 1996, A\&A, in press 